\title{
Scanning tunneling microscopy and tunneling luminescence of the surface of GaN films grown by vapor phase epitaxy
}

\author{
B. Garni, Jian Ma, N. Perkins, Jutong Liu, T. F. Kuech, and M. G. Lagallya) \\ University of Wisconsin-Madison, Madison, Wisconsin 53706
}

(Received 15 November 1995; accepted for publication 2 January 1996)

\begin{abstract}
We report scanning tunneling microscopy (STM) images of surfaces of GaN films and the observation of luminescence from those films induced by highly spatially localized injection of electrons or holes using STM. This combination of scanning tunneling luminescence with STM for $\mathrm{GaN}$ surfaces and the ability to observe both morphology and luminescence in $\mathrm{GaN}$ is the first step to investigate possible correlations between surface morphology and optical properties. (C) 1996 American Institute of Physics. [S0003-6951(96)02110-1]
\end{abstract}

Gallium nitride $(\mathrm{GaN})$ is of great interest because of its potential as a material for the development of light-emitting devices in the blue and ultraviolet spectral region. ${ }^{1-3}$ Extensive investigations have been done on $\mathrm{GaN}$ from different perspectives, most of which have been focused on the exploration of appropriate substrates and growth conditions needed to yield materials suitable for electroluminescent devices. Films of $\mathrm{GaN}$ have been grown on various substrates by several techniques including molecular beam epitaxy (MBE) ${ }^{4}$ and different forms of vapor phase epitaxy (VPE). ${ }^{5,6}$

GaN normally crystallizes with the hexagonal wurtzite structure $(\alpha-\mathrm{GaN}),{ }^{2,7}$ although it can also be synthesized in the cubic zinc-blende phase $(\beta-\mathrm{GaN})$. The techniques utilized in characterizing $\mathrm{GaN}$ include x-ray diffraction, $I-V$ characteristics, photoluminescence, reflection high-energy electron diffraction (RHEED), and Auger electron spectroscopy (AES). For both MBE and VPE growth, we still lack an understanding of the surface morphology of $\mathrm{GaN}$, especially the initial stage of nucleation and its impact on subsequent film growth. Additionally, little is known about the intrinsic properties of $\mathrm{GaN}$ surfaces, restricting our ability to control the surface composition and structure during processing.

Previous studies of $\mathrm{GaN}$ surfaces were limited to the employment of RHEED and $\mathrm{AES}^{8}$ to evaluate long-range order during film growth, and to the study of the effect of sputtering/annealing on $\mathrm{N}$ depletion. Recently, atomic force microscopy (AFM) observations of GaN nucleation layers ${ }^{9}$ and of the influence of open core screw dislocations on the surface morphology of GaN films ${ }^{6}$ have been reported. However, there have as yet been no reports on the use of scanning tunneling microscopy (STM) or scanning tunneling luminescence (STL) measurements for GaN.

In this letter, we report on STM and STL studies of GaN films. Our results demonstrate the use of STM to investigate the surface morphology of GaN. The observed luminescence establishes the use of STL to probe the local surface optical properties of $\mathrm{GaN}$.

STL has recently been proven to be an effective tool in probing local optoelectronic properties. ${ }^{10-16}$ Our STM and STL experiments were performed in a custom-built STM/ STL UHV chamber ${ }^{17}$ with a base pressure of better than

${ }^{\text {a)} E l e c t r o n i c ~ m a i l: ~ l a g a l l y @ e n g r . w i s c . e d u ~}$
$1 \times 10^{-10}$ Torr. STM/STL tips are made from $0.25 \mathrm{~mm}$ diameter tungsten wire by means of electrochemical etching. In STL, the tip is used as a highly localized source of electrons or holes to induce luminescence in the material. The emitted photons are collected by a lens mounted on the microscope base, positioned at $60^{\circ}$ with respect to the sample surface normal, with a solid angle of collection of approximately 1 steradian. The collected light is directed to a lens outside the chamber that focuses it onto a cooled GaAs cathode photomultiplier tube (PMT). The spectral range for our collection/detection apparatus is approximately $320 \mathrm{~nm}-900$ $\mathrm{nm}$, and $\sim 0.8 \%$ (at $360 \mathrm{~nm}$ ) of the total emitted light from the surface can be transmitted to the PMT. Photon counting continues while rastering the tip in a constant-current tunneling mode, allowing both topological and position dependent luminescence data to be acquired simultaneously, giving the capability to correlate luminescence intensity with surface morphology.

GaN samples were grown on (0001) sapphire in a 60 $\mathrm{mm}$ diameter horizontal hot-wall two-temperature-zone halide vapor phase epitaxy (HVPE) reactor. ${ }^{5}$ In the first zone, typically operated around $850{ }^{\circ} \mathrm{C}$, the reaction of MBE-grade 99.9999\% Ga metal with high grade $\mathrm{HCl}$ gas in a $\mathrm{N}_{2}$ carrier gas results in $\mathrm{GaCl}$ and $\mathrm{H}_{2}$ gas reaction products. These products are transported downstream to the second reaction zone, where $\mathrm{NH}_{3}$ is introduced. The reaction of $\mathrm{GaCl}$ with $\mathrm{NH}_{3}$ produces $\mathrm{GaN}$ on the substrate surface, liberating $\mathrm{HCl}$ and $\mathrm{H}_{2}$ as reaction by-products. This second zone is usually operated at $\sim 1040{ }^{\circ} \mathrm{C}$. Films used for this study were produced under a total flow of 10 standard liter per minute, of which 880 standard $\mathrm{cc}$ per minute $(\mathrm{sccm})$ was $\mathrm{NH}_{3}$ and 29.4 sccm was $\mathrm{HCl}$. The rate of film growth is about $1.8 \mu \mathrm{m}$ per minute. Following the growth, the sample is slowly withdrawn from the hot zone under a $\mathrm{NH}_{3}$ atmosphere until the sample has cooled. The reactor is then purged under $\mathrm{N}_{2}$, and the sample is removed for characterization. Epitaxial $\alpha$-GaN films grown in this manner are naturally $n$-type, with a (0001) orientation. The films have a small azimuthal mosaic spread of $\sim \pm 200$ to 450 arcsec. The dislocation density is expected to be high. ${ }^{6}$ The films have a band gap of $3.37 \mathrm{eV}$, as determined by our separate photoluminescence measurements. They exhibit a low reactivity to surface contamination or chemical modification, and are stable enough to be transported from the growth chamber to other chambers 

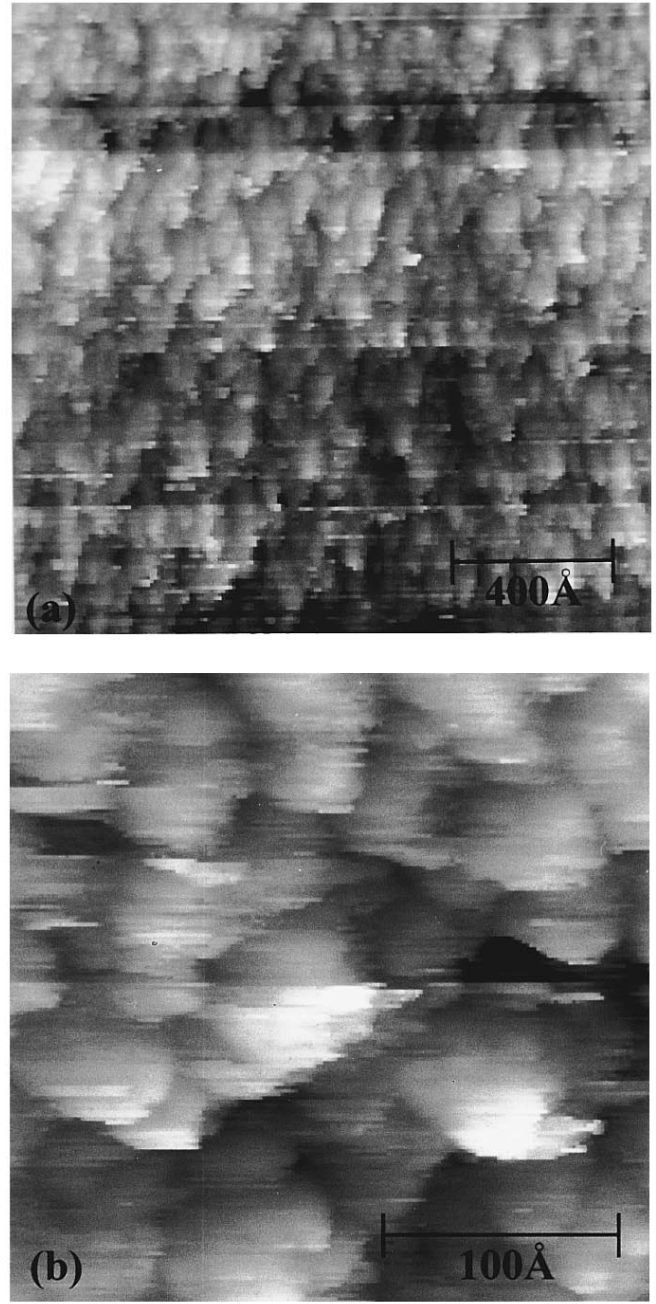

FIG. 1. Scanning tunneling microscopy (STM) images of the surface of VPE grown GaN(0001) films; (a) $1650 \times 1650 \AA$ and (b) $270 \times 270 \AA$. The data were taken with the tip biased at $4.8 \mathrm{~V}$ with respect to the sample with a constant tunneling current of $5 \mathrm{nA}$.

for characterization without the need for extensive surface cleaning. X-ray photoelectron spectroscopy studies performed after such transport show monolayer amounts of $\mathrm{C}$ and $\mathrm{O}$ contamination. ${ }^{18}$

Figure 1 shows $1650 \times 1650 \AA$ (a) and $270 \times 270 \AA$ (b) STM images of the surface of a nominally $\sim 20 \mu \mathrm{m}$ thick $\mathrm{GaN}(0001)$ film. In order to prevent inadvertent modification of the as-grown surface, we performed no in situ cleaning in the STM chamber, relying on the natural passivity of the GaN surface to allow STM imaging. The images in Fig. 1 were taken at a tip bias voltage of $4.8 \mathrm{~V}$ with a tunneling current of $10 \mathrm{nA}$ resulting in the injection of holes into the near-surface region. A high bias voltage is needed for imaging because $\mathrm{GaN}$ has a fairly wide band gap. At low magnification [Fig. 1(a)], we observe an array of relatively flat areas with seemingly random edges. The high-magnification image shown in Fig. 1(b) reveals the roughness of the GaN surface at the near-atomic scale. The bright flat areas correspond to the topmost GaN layers; they have sizes between $\sim 50$ and $120 \AA$. The height between two adjacent terraces varies between 10 and $20 \AA$, which corresponds to 4-8 atomic layers. We obtain similar images with bias voltages of $4.8 \mathrm{~V}, 6.0 \mathrm{~V}$, and $9.0 \mathrm{~V}$, although resolution is reduced at the
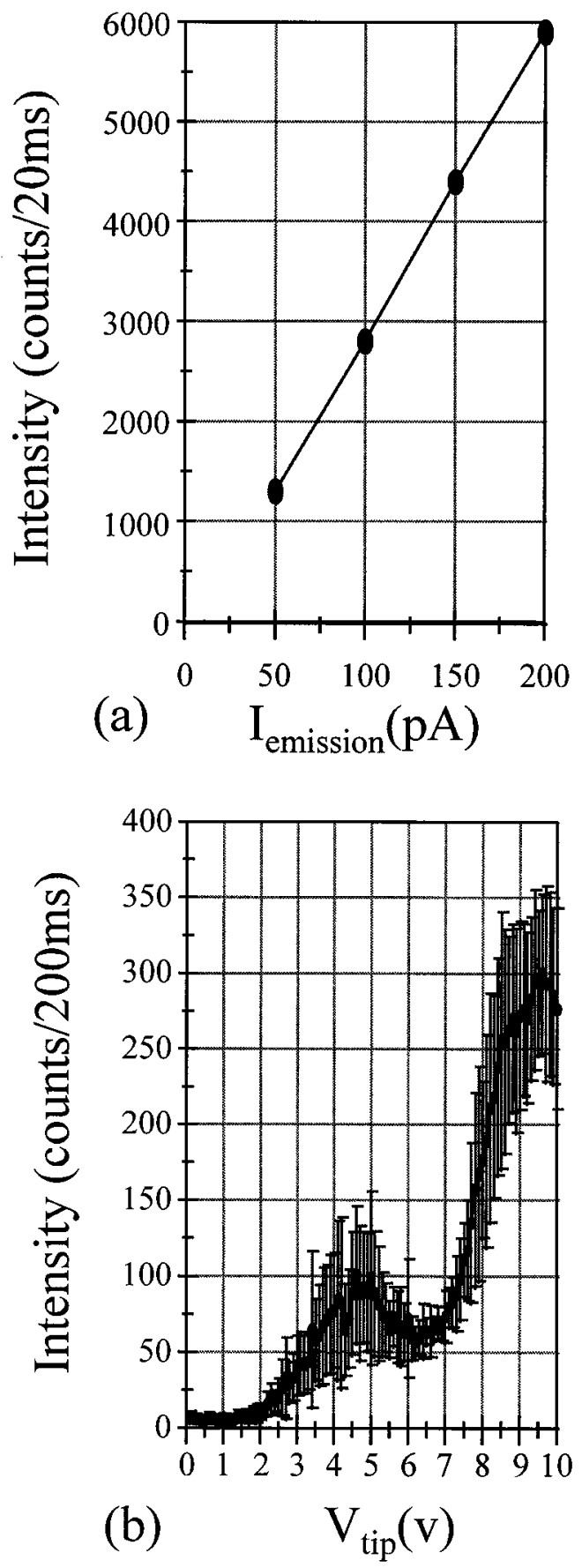

FIG. 2. Spatially localized luminescence intensity from $\mathrm{GaN}(0001)$. (a). Cathodoluminescence using field emission from the STM tip. Dependence of the luminescent intensity on the emission current. The tip is $1 \mu \mathrm{m}$ from the sample and its potential is $\sim-150 \mathrm{~V}$ with respect to the sample. (b). Tunneling mode with hole injection. Dependence of luminescence intensity on the tunneling voltage at a constant tunneling current.

higher bias voltages. At this stage, we have not yet obtained atomic resolution, most probably because of the submonolayer of $\mathrm{C}$ and $\mathrm{O}$ contamination. ${ }^{18}$

Luminescence measurements were made in two ways. In the cathodoluminescence mode, the tip is retracted to $\sim 1$ $\mu \mathrm{m}$ above the sample surface and a voltage of $\sim 150 \mathrm{~V}$ is applied between tip and sample, producing a beam of electrons that easily overcomes the tunneling barrier, but nevertheless is spatially very localized. In the tunneling mode, the tip is in the usual position for topographic measurements, 
$\sim 10 \AA$ from the surface, the bias voltage is of the order of several volts, and electrons tunnel either into or out of the solid. The spatial extent at the point of injection is the same as in STM. Figure 2 shows the luminescent intensity for a $\mathrm{GaN}$ film in both cases. Figure 2(a) shows the dependence of the cathodoluminescent intensity on field emission current. The tip is held at a constant distance. Small changes in the tip-sample voltage (here 143-151 V) change the field emission current. A linear dependence on beam current is observed, as expected. We obtained similar currentluminescence profiles in different regions of the sample. We have so far not measured the spectral distribution of the cathodoluminescence or the tunneling luminescence, but assume that most of the light comes in the band (364 nm) expected for $\mathrm{GaN}$.

Figure 2(b) shows the dependence of the luminescent intensity on the tip voltage in the tunneling mode with the sign of the bias corresponding to hole injection. The measurement is made at fixed lateral position of the tip, but with changing vertical position so as to maintain a constant tunneling current as the voltage is varied. The figure shows an average over 12 measurements; the error bars indicate the variation in the measurements. A simple-minded explanation of the process of the tunneling luminescence may be obtained in the following manner. ${ }^{13}$ The tunneling current will mimic the joint density of states (JDOS) of the GaN valence band and metal tip valence band as electrons tunnel into the metal tip. The holes in $\mathrm{GaN}$ will rapidly thermalize to the top of the valence band. Luminescence occurs through recombination of the holes with electrons at the bottom of the conduction band. With those highly doped $n$-type samples, the Fermi level $\left(E_{f}\right)$ will be very near the conduction band edge, and we should observe a threshold of luminescence at $\sim 3.37 \mathrm{eV}$ (the band gap of GaN). Some luminescence actually begins to appear above $\sim 2 \mathrm{eV}$, suggesting possible deep-level electron trap states in the band gap, in agreement with our photoluminescence measurements. ${ }^{19}$ Near the threshold, the luminescence intensity has a $V^{2}$ dependence, consistent with STL observations on $\mathrm{Al}_{x} \mathrm{Ga}_{1-x} \mathrm{As} .{ }^{13}$ The intensity beyond the threshold increases, but dips slightly near $6.0 \mathrm{eV}$. Calculations of the density of states of $\mathrm{GaN}$, as well as photoemission measurements, ${ }^{8}$ give a shape that has a local minimum $3.5 \mathrm{eV}$ below the top of the valence band. The dip we observe in the luminescence corresponds qualitatively to this dip in the valence band DOS. Beyond about $8.0 \mathrm{eV}$, the intensity shows a monotonic increase, as we begin to reach the field emission region, as for cathodoluminescence.

We have also made two-dimensional maps of the tunneling luminescence. Because the intensity is weak, the images are still quite noisy. Although the luminescence images show features that are reproducible from one scan to the next, we see at this stage no obvious correlation to the morphology observed by the STM. The resolution of STL relative to STM is an open question. Because the mean free path of electrons and holes before recombination may be large, the spatial resolution of STL at first glance is expected to be considerably poorer than that of STM. On the other hand, our calculations show that a high recombination site density at the surface or at defects may greatly enhance the resolution of the luminescence. ${ }^{19}$ At this stage we also do not know to what extent the slight surface contamination affects the luminescence. We are continuing to assess these questions.

In summary, we have succeeded in imaging GaN films with STM and detecting its localized luminescence with STL for the first time. The step morphology of the surface has been observed. The success of STL experiments on GaN opens up the possibility of studying correlations of surface morphology and defect structure with local optical properties of $\mathrm{GaN}$.

This work was supported by ONR, Physics and by NSF Grant No. DMR.91-21074, the Naval Research Laboratory, and the ARPA URI on Visible Light Emitters.

${ }^{1}$ R. F. Davis, Proc. IEEE 79, 702 (1991); Physica B 185, 1 (1993), and references therein.

${ }^{2}$ J. I. Pankove, Mater. Res. Soc. Symp. Proc. 97, 409 (1987).

${ }^{3}$ I. Akasaki, H. Amano, N. Koide, M. Kotaki, and K. Manabe, Physica B 185, 428 (1993)

${ }^{4}$ D. E. Lacklison, J. W. Orton, I. Harrison, T. S. Cheng, L. C. Jekins, C. T. Foxon, and S. E. Hooper, Appl Phys. Lett. 78, 1838 (1995), and references therein.

${ }^{5}$ H. P. Maruska and J. J. Tietjen, Appl. Phys. Lett. 15, 327 (1969).

${ }^{6}$ W. Qian, G. S. Rohrer, M. Skowronski, K. Doverspike, L. B. Rowland, and D. K. Gaskill, Appl. Phys. Lett. 67, 2284 (1995).

${ }^{7}$ D. Elwell, R. S. Feigelson, M. M. Simkins, and W. A. Tiller, J. Cryst. Growth 66, 45 (1984).

${ }^{8}$ R. W. Hunt, L. Vanzetti, T. Castro, K. M. Chen, L. Sorba, P. I. Cohen, W. Gladfelter, J. M. Van Hove, J. N. Kuznia, M. A. Khan, and A. Franciosi, Physica B 185, 415 (1993), and references therein.

${ }^{9}$ D. Kapolnek, X. H. Wu, B. Heying, S. Keller, B. P. Keller, U. K. Mishra, S. P. DenBaars, and J. S. Speck, Appl. Phys. Lett. 67, 1541 (1995).

${ }^{10}$ J. K. Gimzewski, B. Reihl, J. H. Coombs, and R. R. Schlittler, Z. Phys. B 72, 497 (1988).

${ }^{11}$ D. L. Abraham, A. Veider, Ch. Schönenberger, H. P. Meier, D. J. Arent, and S. F. Alvarado, Appl. Phys. Lett. 56, 1564 (1990).

${ }^{12}$ S. F. Alvarado, Ph. Renaud, D. L. Abraham, Ch. Schönenberger, D. J. Arent, and H. P. Meier, J. Vac. Sci. Technol. B 9, 409 (1991).

${ }^{13}$ P. Renauld and S. F. Alvarado, Phys. Rev. B 44, 6340 (1991).

${ }^{14}$ S. Ushioda, Solid State Commun. 84, 173 (1992).

${ }^{15}$ L. Samuelson, A. Gustafsson, J. Lindahl, L. Montelius, M.-E. Pistol, J.-O. Malm, G. Vermeire, and P. Demeester, J. Vac. Sci. Technol. B 12, 2521 (1994).

${ }^{16}$ V. Sivel, R. Coratger, F. Ajustron, and J. Beauvillain, Phys. Rev. B 51, 14598 (1995).

${ }^{17}$ B. Garni, Ph.D. thesis, University of Wisconsin-Madison, 1996 (unpublished).

${ }^{18}$ Jian Ma, B. Garni, and M. G. Lagally (unpublished).

${ }^{19} \mathrm{~J}$. Liu and T. Kuech (unpublished). 